\title{
Uncommon cancers of the small intestine, appendix and colon: An analysis of SEER 1973-2004, and current diagnosis and therapy
}

\author{
BJORN I. GUSTAFSSON ${ }^{1-3}$, LUNA SIDDIQUE ${ }^{1}$, ANTHONY CHAN ${ }^{1}$, MANKU DONG $^{1,4}$, \\ IGNAT DROZDOV ${ }^{1}$, MARK KIDD ${ }^{1}$ and IRVIN M. MODLIN ${ }^{1}$ \\ ${ }^{1}$ Department of Surgery, Yale University School of Medicine, New Haven, CT 06520-8062, USA; \\ ${ }^{2}$ Department of Gastroenterology, St Olavs Hospital HF, Trondheim University Hospital, \\ ${ }^{3}$ Department of Cancer Research and Molecular Medicine, Faculty of Medicine, \\ Norwegian University of Science and Technology, N-7006 Trondheim, Norway; \\ ${ }^{4}$ PLA 306th Hospital, Center of General Surgery, Beijing 100101, P.R. China
}

Received May 27, 2008; Accepted August 18, 2008

DOI: 10.3892/ijo_00000101

\begin{abstract}
Analysis of the Surveillance, Epidemiology and End Results (SEER) registry indicates that more than $20 \%$ of all cancers are located in the gastrointestinal (GI) tract. Although colon adenocarcinomas constitute $\sim 90 \%$ of all malignant intestinal neoplasia, the remaining $10 \%$ of tumors in the small intestine (SI), appendix and colon are clinically relevant since their late presentation due to a paucity of overt symptoms culminates in a high mortality rate despite the fact that many such lesions are not intrinsically aggressive neoplasia. Thus, neuroendocrine tumors (NETs), adenocarcinomas (except for colonic), lymphomas, sarcomas and GI stromal tumors (GISTs) of the SI, appendix and colon, while relatively rare, represent an under-recognized and underserved group of lesions. According to the SEER registry 1973-2004, the incidence/100,000 of sarcomas has remained unchanged, while NETs, adenocarcinomas (except colon), and lymphomas have increased 2.9-, 1.6-, and 2.0-fold, respectively. This may, at least partly, reflect the development of more sophisticated diagnostic techniques including high resolution CT and MRI, capsule endoscopy and somatostatin scintigraphy for NETs. Although the development of specific targeted therapies such as tyrosine kinase inhibitors (TKIs) for GISTs and somatostatin analogs for NETs have improved prognosis, early detection remains the critical variable in
\end{abstract}

Correspondence to: Dr Irvin M. Modlin, Department of Gastroenterological Surgery, Yale University School of Medicine, 333 Cedar Street, P.O. Box 208062, New Haven, CT 06520-8062, USA

E-mail: imodlin@optonline.net

Key words: adenocarcinoma, carcinoid, epidemiology, lymphoma, neuroendocrine, sarcoma, SEER, tumor determining outcome. The overall 5-year survival rates have remained relatively unchanged over time (1973-1999), or are only improved marginally for some subgroups. We present an overview of the epidemiology of these uncommon cancers, and address their clinical behavior, and current diagnostic and therapeutic options.

\section{Contents}

1. Introduction

2. Statistics

3. Specific neoplasms

4. Diagnosis

5. Treatment

6. Summary

\section{Introduction}

Analysis of the SEER data base from 1973-2004 containing $3,104,173$ cancers, revealed that $20.4 \%$ of all cancers are located in the gastrointestinal (GI) system (1). The SEER program of the National Cancer Institute currently covers 18 diverse geographical areas in the US comprising $~ 26 \%$ of the population and generating a sample predicted to provide an acceptable cross-section of cancer epidemiology in North America (1). While the SI accounts for $80 \%$ of the total intestinal length and $90 \%$ of its absorptive surface, SI cancers account for just $3.1 \%$ of all intestinal cancers. This is in stark contrast to the colon and rectum which are $\sim 20 \%$ of overall intestinal length but are the site of 68.9 and $27.3 \%$ of lesions while appendiceal cancers represent $0.7 \%$. The reason for the significant over-representation of colorectal adenocarcinomas is unknown. Putative etiologic factors including $\mathrm{pH}$ differences, shorter SI transit time with reduced contact time of luminal carcinogens, increased cancer risk in colon constipation, lower bacterial load in the SI, and a higher mucosal renewal rate 
(enterocyte proliferation index) in the SI with shedding of cells prior to euplastic transformation have all variously been proposed $(2,3)$.

Of the malignant SI neoplasms, NETs, comprise $36.5 \%$, adenocarcinomas $30.9 \%$, sarcomas and GISTs $10.0 \%$, lymphomas $18.7 \%$, and miscellaneous and non-specified neoplasia $3.9 \%$ (Fig. 1). Appendiceal include NETs 31.7\%, adenocarcinomas $65.4 \%$, sarcomas and GISTs $<1 \%$, lymphomas $1.7 \%$, and miscellaneous and non-specified $1.1 \%$. Colon tumors include NETs $0.6 \%$, adenocarcinomas $93.0 \%$, sarcomas and GISTs $0.1 \%$, and lymphomas $0.4 \%$, and miscellaneous and non-specified 5.9\%. Metastatic disease to the bowel is rare, mainly confined to the SI and most commonly reflects hematological spread from melanoma, lung, breast, and renal cancer, or via the lymphatic intraperitoneal route from neoplasia including ovarian, pancreatic, and gastric carcinomas.

The abdomen's repertoire of symptoms is limited (pain, nausea, diarrhea, vomiting, constipation and bloating), and the SI exhibits symptomatology that is vague and non-specific. Thus, symptoms may be present for many years prior to a diagnosis being established by a variety of biochemical, radiological, and endoscopic techniques, either alone or in combination. Consequently, the cancer is often at an advanced stage and hence exhibits a poor outcome. In general, the primary treatment is always complete surgical resection whenever possible. However, dependent on factors such as histological subtype and extent of metastatic spread, a variety of chemotherapeutic and biotherapeutic agents and radiotherapy and cytoreductive regimens may require consideration. Prognosis is directly related to both the histological subtype of the tumor, local extent of the disease and the degree of metastatic spread.

We have interrogated the SEER data base to present a contemporary overview of the epidemiology of these uncommon SI, appendix and colon tumors with the object of addressing their clinical behavior, and the current diagnostic and therapeutic options necessary to delineate the current status of their management.

\section{Statistics}

All incidence rates (per 100,000 population per year) extracted from 1973-2004 were age-adjusted using the US 2000 standard population and are presented in terms of site, gender and race. The observed 5-year survival was calculated using the actuarial method (4). Linear regression curves were created with the GraphPad Prism 4 software.

\section{Specific neoplasms}

Neuroendocrine tumors (NETs, carcinoids). Cells of the diffuse neuroendocrine cell system (DNES) are dispersed throughout the GI, bronchopulmonary, and urogenital systems as either single cells or clusters. Tumors of the DNES are often referred to as carcinoids in deference to the original report presented by Oberndorfer in 1907, although this group colloquation has resulted in preserving the erroneous and archaic concept that they represent one uniform tumor type (5). In actuality, each tumor is derived from an individual specific cell type (enterochromaffin, enterochromaffin-like, gastrin, etc.) and exhibits distinct disparate clinical and biological behavior.

SI NETs. SI-NETs comprise $24.3 \%$ of all NETs (6). Their incidence was 0.92 in 2004 (duodenal 0.21 , jejunal 0.04, ileal 0.41, unspecified SI 0.26). This represents a 4.4-fold increased incidence since 1973 (Fig. 2A). The majority $(\sim 60 \%)$ of SI-NETs are diagnosed when the disease is no longer localized and metastatic spread to the liver is present. An early diagnosis is often delayed ( $\sim 5$ years) as most SI tumors are initially small and asymptomatic, or misdiagnosed as more prosaic diseases such as functional bowel disorder or an allergy. However, as the tumor evolves in size, obstruction, perforation, and bleeding can arise due to local tumor mass effects or tumor-induced fibrosis, and may result in presentation as an 'acute abdomen' in $~ 5 \%$ of SI-NETs (6). In the event of liver metastases, bioactive tumor products may enter the systemic circulation, bypassing hepatic inactivation and engender the 'carcinoid syndrome'. Classically, this consists of protean symptomatology including episodic skin flushing, diarrhea, bronchoconstriction, sweating, and abdominal cramping. As many as $50 \%$ of individuals may also, in addition, exhibit cardiac valvular disease (7). Overall, $33 \%$ of lesions are multicentric and $\sim 18 \%$ of individuals with jejuno-ileal NETs present with the carcinoid syndrome. The overall 5-year survival rate of patients with SI-NETs 1973-1999 was $64.1 \%$, but has increased by a rate of $\sim 0.5 \%$ / year and reached $71.8 \%$ in 1999 (Fig. 2B). The 5-year survival in those with additional hepatic metastasis is only $40 \%$ (8), and the therapy of distant spread is therefore a critical management issue.

Five types of duodenal NETs can currently be distinguished: i) duodenal gastrinomas, $( \pm 65 \%$ of duodenal NETs $)$, ii) somatostatinomas (SSTomas) $(15 \%)$, iii) non-functioning, iv) poorly differentiated, predominantly ampullary NE carcinomas, and v) duodenal gangliocytic paragangliomas. Gastrinomas are located predominantly in part I or II of the duodenum, and are usually $<1 \mathrm{~cm}$. Despite their small size metastases are often evident in regional lymph nodes (LN). Such metastases may be larger than the primary and have, in some instances, erroneously been considered pancreatic endocrine tumors, especially if in close proximity to the pancreas (9). Duodenal SSTomas preferentially occur is in the region of the papilla of Vater or periampullary area and if the muscularis propria is invaded, it is likely that paraduodenal LN metastases will be present (10). Non-functioning duodenal NETs have a prognosis much more favorable than gastrinomas or ampullary SSTomas with metastases only evident once the tumor has extended beyond the submucosa (11).

Poorly differentiated duodenal carcinomas occur primarily in the region of the papilla of Vater, are usually hormonally inactive and exhibit advanced metastasis into the regional LN and the liver. Duodenal gangliocytic paragangliomas occur in the periampullary area and although often $>2 \mathrm{~cm}$ with invasion of the muscularis propria, they generally exhibit a benign course. In a study of 89 patients with duodenal NETs, the overall 5-year survival was $60 \%$ (12).

Meckel's diverticulum is an anatomic variant representing a remnant of a patent vitelline duct and is found on the antimesenteric side of the ileum, within $\sim 60 \mathrm{~cm}$ of the terminal 

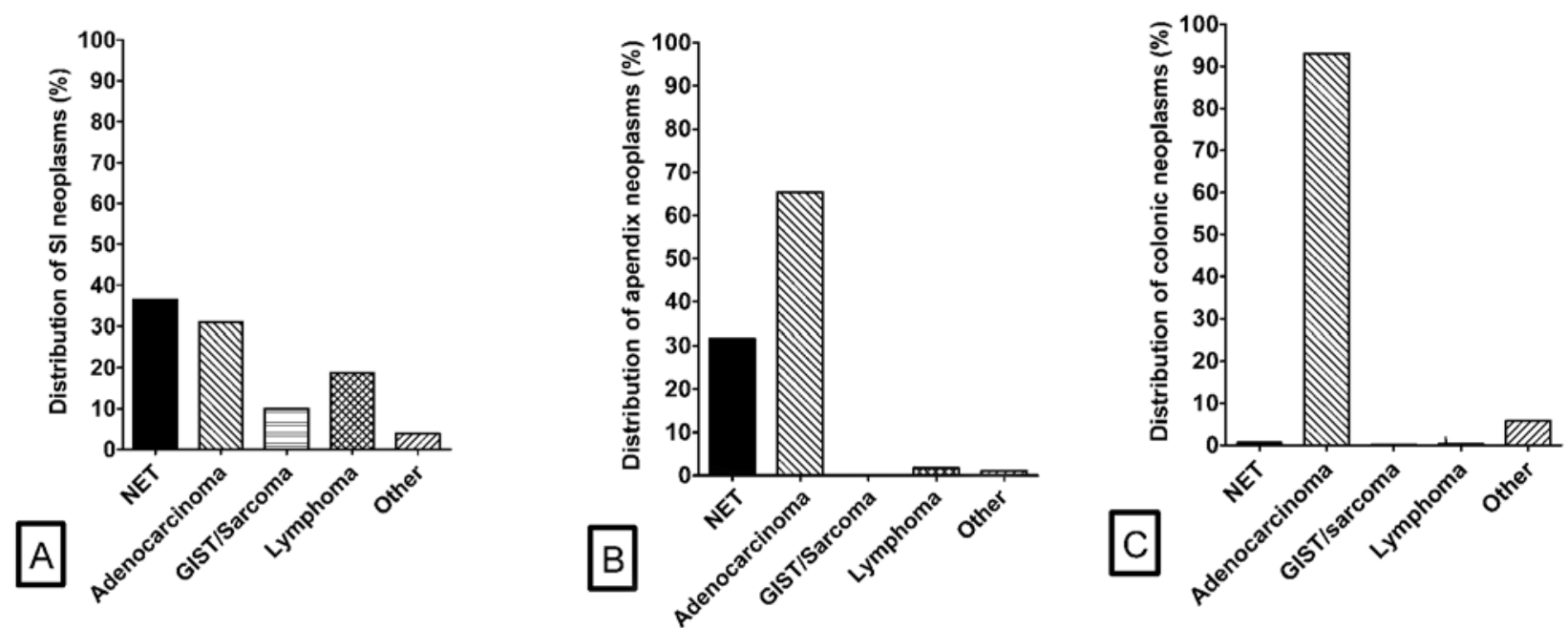

Figure 1. Distribution of: A, small intestinal (SI) $(n=12,516)$; B, appendix ( $n=2,791)$; and C, colon cancers ( $n=276,048)$, registered in the SEER database 1973-2004.
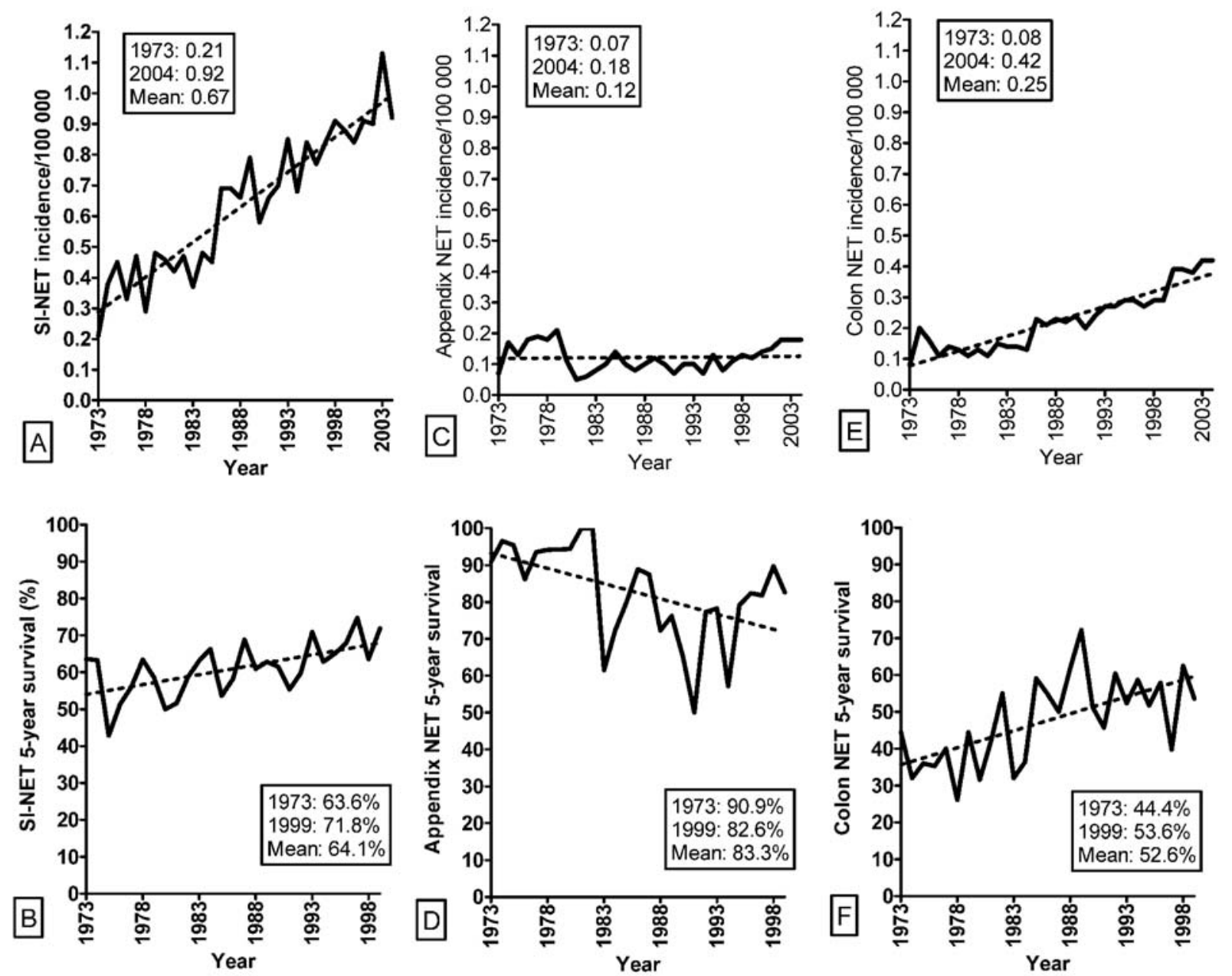

Figure 2. A and B, Incidence and 5-year survival of small intestinal (SI)-NETs. C and D, Incidence and 5-year survival of appendix NETs. E and F, Incidence and 5-year survival of colon NETs. Dotted lines represent regression curves.

ileum, in $\leq 2 \%$ of the population. NETs occurring within a Meckel's diverticulum are usually gastric enterochromaffin-like cell (ECL) lesions related to the aberrant gastric mucosa often found in these embryological remnants. Tumors associated with Meckel's diverticula are reported at a rate of $3.2 \%$, of which NETs comprise one-third, and the rest are sarcomas, 
adenocarcinomas, mesenchymal tumors, melanomas and lymphomas (13). The clinical presentation and prognosis have been evaluated and are similar to that of SI-NETs (14).

Appendix NETs. Appendix NETs account for $4.7 \%$ of all NETs (6). The incidence has been stable between 0.1-0.2 over the past 30 years (Fig. 2C). They are usually small, clinically apparently benign lesions and are most often discovered as an incidental finding during appendectomy. The carcinoid syndrome is rare. Although in most instances, the tumors are derived from the enterochromaffin cell, there exist a number of subtypes including goblet cell carcinoid (GCC, also known as adenocarcinoid), composite carcinoid and atypical carcinoid tumor. GCC accounted for $46.2 \%$ of all appendix NETs (Table I). The mean age of presentation is 58.9 years, with an equal representation in both males and females (15). GCC most commonly manifests as acute appendicitis, however, presentation can vary from asymptomatic (identified incidentally at laparoscopy or unrelated surgery) to complete bowel obstruction. The 5-year survival rates for those with localized NETs, regional spread and distant metastases were 94,84 and $26 \%$ respectively, with an overall survival rate of $83.3 \%$ (Fig. 2D). The 5-year survival rates according to the major histological subtypes were carcinoid tumor malignant $79.6 \%$, GCC 78.6\%, and composite NET $49.2 \%$ (1).

Colon NETs. Colon NETs (rectum excluded) account for $8.8 \%$ of all NETs and occur most frequently $(53 \%)$ in the cecum (6). The incidence has increased 5.2-fold (0.08-0.42) (Fig. 2E). The carcinoid syndrome is very uncommon $(<5 \%)$ and the tumor is often only identified when it presents with bleeding, pain or obstruction, or incidentally at routine endoscopy or surgery. Colon NETs exhibit the worst prognosis of GI-NETs, with an overall 5-year survival of 52.6\%, although this figure has increased from $44.4 \%$ in 1973 to $53.6 \%$ in 2004 (Fig. 2F).

\section{Adenocarcinoma}

SI adenocarcinomas. Adenocarcinomas account for $30.9 \%$ of all SI tumors (Fig. 1). The incidence has increased from 0.49 to 0.66 (1973-2004) (Fig. 3A). They are most commonly found in the duodenum $(\sim 50 \%)$, followed by the jejunum $(\sim 20 \%)$ and ileum $(\sim 15 \%)$. The reasons for this anatomic spread are unclear, though the proliferative and possible carcinogenic effects of bile have been suggested as possibilities (16). The peak incidence occurs in the sixth decade with a male predominance of $\sim 60 \%$ (17). Ulceration is common, which may lead to occult GI bleeding or chronic anemia. Duodenal adenocarcinomas present with gastric outlet obstruction, while those with more distal lesions tend to have cramping abdominal pain. In a series of 217 patients with SI adenocarcinoma, the most common presenting symptoms were abdominal pain $(67 \%)$, obstruction $(40 \%)$, and bleeding (24\%) (18).

Of note is the evidence of an increased relationship with both Crohn's disease (x12) and celiac disease ( $x 35)$ $(19,20)$. Cancers typically occur in the regions affected by the inflammation and although no mechanistic basis has been elucidated, the relationship between an altered local cytokine milieu and immune perturbations is likely contributory (21). Adenocarcinomas may also arise as a component of polypoid conditions including familial adenomatous polyposis and Peutz-Jeghers syndrome. In such groups, surveillance with capsule endoscopy is recommended (22). The overall 5-year survival is $20 \%$, and has not improved during the study period (Fig. 3B). The 5-year survival for subtypes including mucinous adenocarcinoma and signet-ring cell carcinoma were 21.4 and $6.7 \%$ respectively (1).

Appendix adenocarcinomas. Adenocarcinomas account for $65.4 \%$ of appendiceal cancers (Fig. 1). The incidence has increased 2.6-fold (0.11-0.29) (Fig. 3C). The majority ( 80\%) present with symptoms of acute appendicitis at a mean age of 52 years (23). Due to the thin muscular wall of the appendix, $\sim 50 \%$ perforate when the lumen becomes obstructed by tumor growth resulting in dissemination of tumor cells throughout the abdomen and pelvis which may culminate in the development of mucinous ascites or pseudomyxoma peritonei. The overall 5 -year survival of appendiceal adenocarcinomas was $46.2 \%$, and it is noteworthy that the 5-year survival has decreased from 66.7-43.4\% (1973-1999) (Fig. 3D). The reported 5-year survival for subgroups was: adenocarcinoma $47.9 \%$, mucinous adenocarcinoma $47.7 \%$, mucinous cystadenocarcinoma $59.0 \%$, and signet ring cell carcinoma $20.3 \%$ (1).

Colon adenocarcinomas. Colon adenocarcinomas do not fall within the primary goal of this study but some epidemiological data are presented to facilitate comparison and provide perspective. Adenocarcinomas represent $93.0 \%$ of all colon tumors, with an incidence of 29.1 in 2004 (Fig. 3E). Of note is that the incidence peaked in 1985 (40.6) and has thereafter decreased steadily. A probable explanation for the decreasing incidence is the increasing use of colonoscopy and introduction of population-based colonoscopy screening programs. This observation is in direct contradistinction for both the poor prognosis and lack of improvement in prognosis for SI and appendix adenocarcinomas respectively. Indeed, the 5-year survival for colon adenocarcinomas is improving (40.0$51.1 \%$ ), (Fig. 3F) indicating that early detection and elimination of precursor lesions (polyps) probably contribute to the positive trend.

Lymphomas. GI tract lymphomas may arise in four compartments; organized lymphoid tissue (e.g., Peyer's patches in the terminal ileum), the lamina propria, intraepithelial lymphocytes, and mesenteric lymph nodes. Broadly speaking, lymphoma is categorized as Hodgkin's lymphoma (HL) and non-Hodgkin's lymphoma (NHL). NHL are staged and classified into specific NHL subtypes (embracing all other types of lymphoma, including diffuse, large B-cell, follicular, Burkitt lymphoma, small B-cell lymphoma, marginal zone B-cell lymphoma, Mantle cell lymphoma, T-cell lymphoma, and lymphoblastic lymphoma (Table I).

The incidence of SI and colon lymphomas has increased from 0.22 to 0.35 and 0.1 to 0.21 , respectively (Fig. $4 \mathrm{~A}$ and $\mathrm{E}$ ). Lymphomas of the appendix were rare representing $1.7 \%$ of all appendix tumors (Fig. 1). Primary HL of the GI tract is extremely rare and many regard the existence of this entity with skepticism.

SI NHL. SI lymphomas are most commonly of diffuse large B-cell type (46.7\%) (Table I). B-cell lymphomas usually exhibit histological features similar to gastric mucosaassociated lymphoid tissue (MALT) lymphomas. While 
Table I. Distribution of 291,355 tumors registered in SEER 1973-2004.

\begin{tabular}{|c|c|c|c|c|c|c|}
\hline & \multicolumn{2}{|c|}{ Small bowel } & \multicolumn{2}{|c|}{$\begin{array}{l}\text { Location } \\
\text { Appendix }\end{array}$} & \multicolumn{2}{|c|}{ Colon } \\
\hline & $\mathrm{n}$ & $\%$ & $\mathrm{~N}$ & $\%$ & $\mathrm{n}$ & $\%$ \\
\hline \multicolumn{7}{|l|}{ Neuroendocrine tumors, $n=7,069$} \\
\hline Carcinoid tumor malignant & 4,240 & 92.9 & 413 & 46.7 & 1,247 & 76.9 \\
\hline Neuroendocrine carcinoma & 205 & 4.5 & 11 & 1.2 & 271 & 16.7 \\
\hline Enterochromaffin cell carcinoid & 64 & 1.4 & 22 & 2.5 & 19 & 1.1 \\
\hline Goblet cell carcinoid (adenocarcinoid) & 23 & 0.5 & 408 & 46.2 & 47 & 2.9 \\
\hline Composite carcinoid & 14 & 0.3 & 30 & 3.4 & 30 & 1.9 \\
\hline Gastrinoma, malignant & 14 & 0.3 & - & - & - & - \\
\hline Other & 4 & 0.1 & 0 & 0 & 7 & 0.4 \\
\hline Total and $\%$ of all locations & 4,564 & $(64.6)$ & 884 & $(12.5)$ & 1,621 & $(22.9)$ \\
\hline \multicolumn{7}{|l|}{ Adenocarcinoma, $n=262,294$} \\
\hline Adenocarcinoma, NOS & 2,970 & 76.7 & 627 & 34.4 & 189,594 & 73.9 \\
\hline Mucinous & 435 & 11.2 & 767 & 42.0 & 28,501 & 11.1 \\
\hline In villous adenoma & 216 & 5.6 & 77 & 4.7 & 20,440 & 7.9 \\
\hline Signet ring cell & 88 & 2.3 & 127 & 7.0 & 1,657 & 0.6 \\
\hline Papillary, NOS & 64 & 1.6 & 16 & 0.9 & 1,144 & 0.4 \\
\hline In adenomatous polyposis coli & 40 & 1.0 & - & - & 13,942 & 5.4 \\
\hline Intestinal type & 26 & 0.7 & - & - & 189 & 0.1 \\
\hline Villous adenocarcinoma & 10 & 0.3 & - & - & 408 & 0.2 \\
\hline Cystadenocarcinoma & & & 179 & 9.8 & 8 & 0.003 \\
\hline Other & 23 & 0.6 & 22 & 1.2 & 714 & 0.3 \\
\hline Total and $\%$ of all locations & 3,872 & $(1.5)$ & 1,825 & $(0.7)$ & 256,597 & $(97.8)$ \\
\hline \multicolumn{7}{|l|}{ GIST/sarcoma, $\mathrm{n}=1,538$} \\
\hline Leiomyosarcoma, NOS & 672 & 53.5 & - & - & 137 & 49.5 \\
\hline Gastrointestinal stromal sarcoma & 321 & 25.6 & - & - & 42 & 15.2 \\
\hline Sarcoma, NOS & 64 & 5.1 & - & - & 16 & 5.8 \\
\hline Epithelioid leiomyosarcoma & 52 & 4.1 & - & - & 14 & 5.1 \\
\hline Kaposis sarcoma & 43 & 3.4 & 6 & - & 27 & 9.7 \\
\hline Spindle cell & 29 & 2.3 & - & - & - & \\
\hline Endometrial stromal sarcoma & 13 & 1.0 & - & - & - & \\
\hline Other & 61 & 4.8 & - & - & 41 & 14.8 \\
\hline Total and $\%$ of all locations & 1,255 & $(81.6)$ & 6 & $(0.4)$ & 277 & $(18.0)$ \\
\hline \multicolumn{7}{|l|}{ Non-Hodgkin's lymphoma, $\mathrm{n}=3,597$} \\
\hline Large B-cell, diffuse & 1,096 & 46.7 & 11 & 23.4 & 659 & 54.7 \\
\hline Follicular & 407 & 17.7 & - & - & 66 & 5.5 \\
\hline Grade 1 & $(215)$ & $(9.2)$ & - & - & $(24)$ & $(2.0)$ \\
\hline Grade 2 & (78) & $(3.3)$ & - & - & (13) & $(1.1)$ \\
\hline Grade 3 & (43) & $(1.8)$ & - & - & (14) & $(1.2)$ \\
\hline NOS & (71) & $(3.0)$ & - & - & $(15)$ & $(1.2)$ \\
\hline Malignant, NOS & 395 & 16.8 & - & - & 207 & 17.2 \\
\hline Small B lymphocytic, NOS & 95 & 4.1 & - & - & 44 & 3.7 \\
\hline Burkitt, NOS & 94 & 4.0 & - & - & 50 & 4.1 \\
\hline Marginal zone B-cell, NOS & 81 & 3.5 & - & - & 74 & 6.1 \\
\hline Mixed small and large cell, diffuse & 65 & 2.8 & - & - & 24 & 2.0 \\
\hline T-cell & 32 & 1.4 & - & - & - & - \\
\hline Mantle cell & 29 & 1.2 & - & - & 47 & 3.9 \\
\hline Anaplastic Ig. Cell, T-cell and Null cell type & 19 & 0.8 & - & - & - & - \\
\hline Lymphoplamacytic & 15 & 0.6 & - & - & - & - \\
\hline Other & 17 & 0.7 & 36 & 76.6 & 34 & 2.8 \\
\hline Total and $\%$ of all locations & 2,345 & $(65.2)$ & 47 & $(1.3)$ & 1,205 & $(33.5)$ \\
\hline Hodgkin's lymphoma, $\mathrm{n}=16$ & 11 & $(68.7)$ & 1 & $(6.2)$ & 4 & $(25)$ \\
\hline Miscellaneous tumors, $n=16,841$ & 469 & $(2.8)$ & 28 & $(0.2)$ & 16,344 & $(97.0)$ \\
\hline Total and $\%$ of tumors at location & 12,516 & $(4.3)$ & 2,791 & $(1.0)$ & 276,048 & $(94.7)$ \\
\hline
\end{tabular}



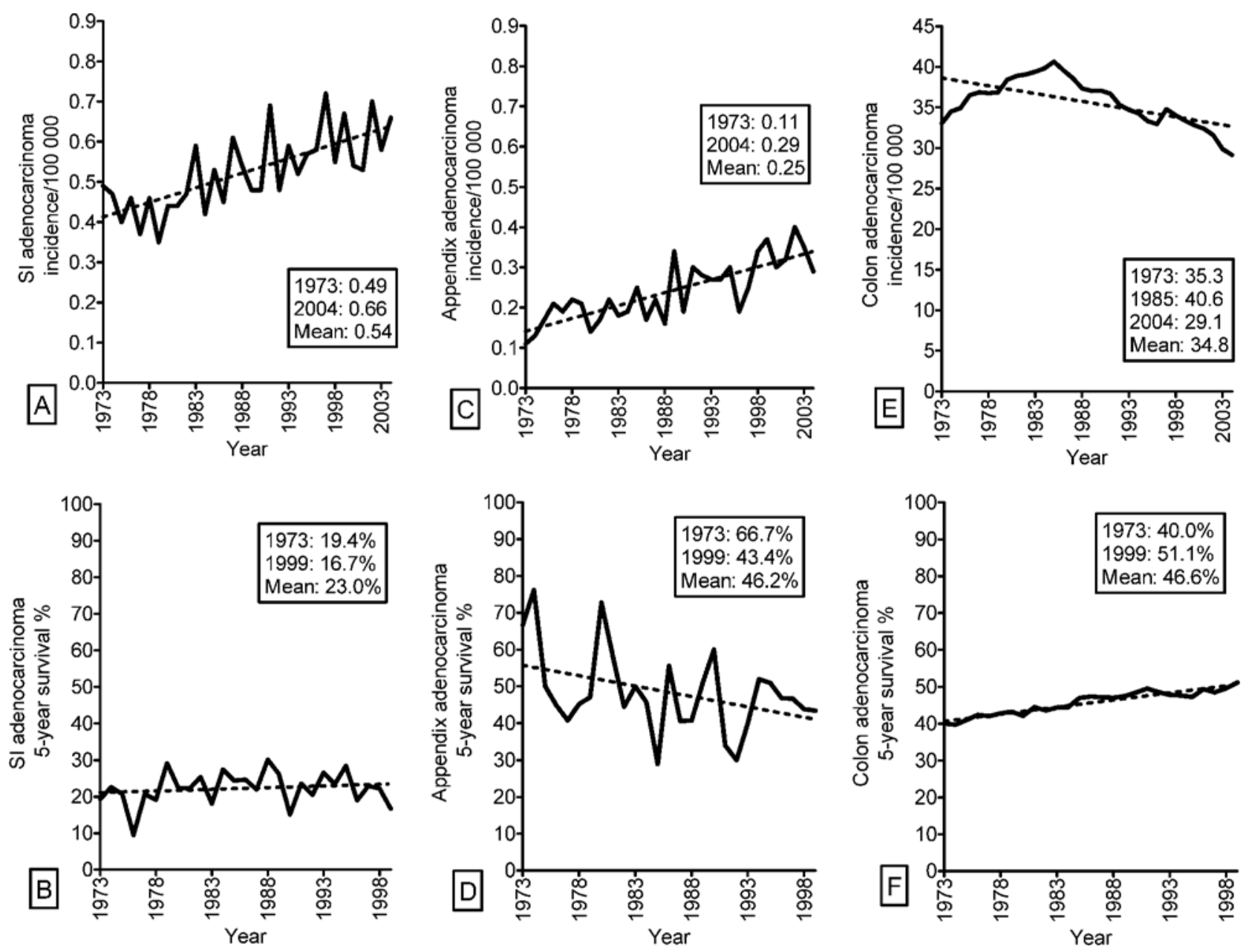

Figure 3. A and B, Incidence and 5-year survival of small intestinal (SI) adenocarcinomas. C and D, Incidence and 5-year survival of appendix adenocarcinomas. $\mathrm{E}$ and $\mathrm{F}$, Incidence and 5-year survival of colon adenocarcinomas. Dotted lines represent regression curves.

gastric MALT lymphomas are associated with Helicobacter infection, no specific pathogen has been linked to the tumor equivalent in the SI. Lymph node equivalent B-cell (follicular) lymphoma represents $17.7 \%$ and in $\sim 50 \%$ present as numerous bowel polyps (24). Burkitt lymphoma is a rare (4.0\%), EpsteinBarr virus (EBV) and human immunodeficiency virus (HIV) associated aggressive tumor (25). It most commonly occurs in the ileo-cecal region. The most common presentations of SI lymphoma are abdominal pain (71\%), ileus (38\%), weight loss $(29 \%)$, bleeding $(21 \%)$, perforation (16\%) and a palpable mass (12\%) (26).

Enteropathy (celiac disease) type T-cell lymphoma (ETL) is postulated to arise from intestinal intraepithelial T-cells. ETL can complicate chronic celiac disease, but usually follows a short history of adult celiac disease and/or dermatitis herpetiformis. ETL typically occurs in the sixth and seventh decades, but may also occur in younger individuals (27). In the SEER database, ETL is exceedingly rare, accounting for only $1.4 \%$ of SI lymphomas. Typically it manifests as the reappearance of abdominal pain and malabsorption symptoms in a celiac patient previously responding to a gluten-free diet. Lymphomas originating in the mantle and marginal zones of organized lymphoid nodules are also extremely rare (1.2 and $3.5 \%$ respectively of SI lymphomas). The 5-year survival was 43.3 in $1973,51.9 \%$ in 1999, and overall between 1973-1999, 52.0\% (Fig. 4B). Of the NHL subtypes, follicular grade 1 showed the best prognosis with a 5-year survival of $83.7 \%$, and mature $\mathrm{T}$ cell the worst $15.6 \%$ (1).

Colon NHL. Lymphomas of the colon account for $\sim 10 \%$ of GI lymphomas and most commonly $(\sim 73 \%)$ occur in the cecum (28). The majority (54.7\%) of colonic lymphomas are of the large B-cell, diffuse type. The mean age at diagnosis is $\sim 60$ years, and they commonly present with abdominal pain, anorexia and an abdominal mass (29). Inflammatory bowel disease, HIV/AIDS and immunosuppression are all associated with an increased risk in the development of colon lymphoma (29). Historically the most common types of aggressive colon lymphomas have been diffuse large B-cell lymphoma and Burkitt lymphoma (30), however, more recently an increase of other histological subtypes, including peripheral T-cell, MALT, Hodgkin's and Mantle cell lymphomas has been noted (31). This increase most likely represents improvements in pathological and molecular diagnostic techniques. The overall 5-year survival was $47.9 \%$ for colon lymphomas (Fig. 4F).

Sarcomas and GISTs. GISTs are specific, generally KIT (CD117)-positive, mesenchymal tumors previously referred 

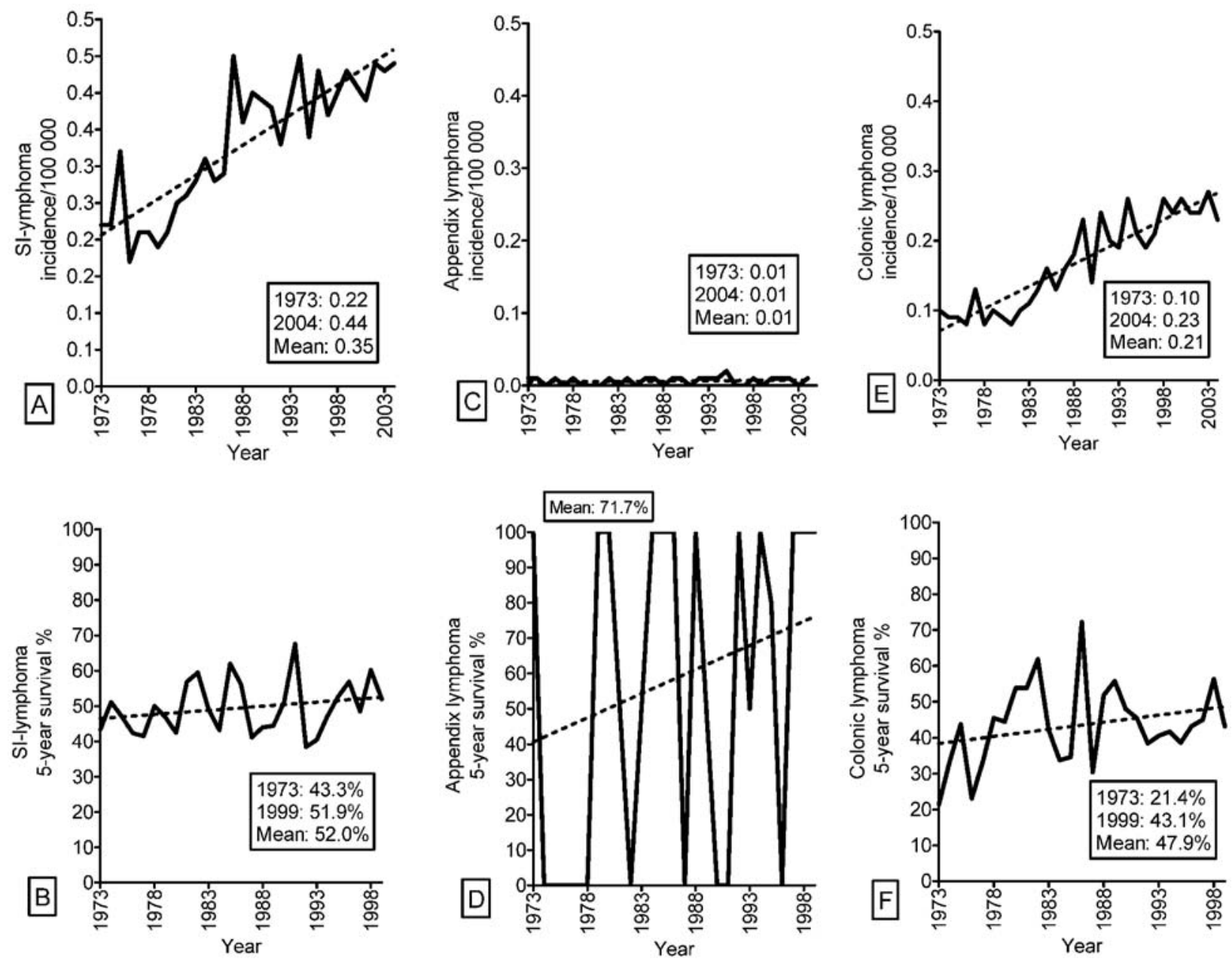

Figure 4. A and B, Incidence and 5-year survival of small intestinal (SI) lymphomas. C and D, Incidence and 5-year survival of appendix lymphomas. Due to the low number of reported appendix lymphomas, the year to year variation is very big. E and F, Incidence and 5-year survival of colon lymphomas. Dotted lines represent regression curves.

to as smooth muscle tumors: leiomyomas, leiomyoblastomas, and malignant leiomyosarcomas. They are considered to originate from the interstitial cells of Cajal or related stem cells, which almost always express KIT1, a tyrosine kinase encoded by the oncogene KIT2 (32). The incidence of sarcomas has remained stable, 0.18 and 0.04 for SI and colon, respectively (Fig. 5). Sarcomas and GISTs account for $10.0 \%$ of SI tumors, $0.1 \%$ of colon malignancies and are almost non-existent in the appendix with only 6 reported cases of Kaposi's sarcoma in SEER. They most commonly present with acute or chronic GI bleeding, but occasionally perforation and GI obstruction may lead to an acute abdomen. In a series of 288 GISTs, $69 \%$ were detected due to symptoms (median age of 68$), 21 \%$ were incidental findings at surgery, and $10 \%$ were found at autopsy (33). The incidence of other neoplasia including colorectal, urinary tract, pancreatic, breast and gastric, with GISTs is reported to be as high as $27 \%(1,33)$. Correct identification of GISTs is critical as effective, specific targeted treatments including tyrosine kinase inhibitors (TKIs) are available. Following the introduction of Imatinib (a TKI) in 2000, there has been a significant increase in survival (34). The 5-year survival rate of SI and colonic GISTs were 39.8 and $34.2 \%$ respectively (Fig. 5).

\section{Diagnosis}

Biochemistry. Laboratory investigations are often inconclusive. Elevated 24-h urinary 5-hydroxyindoleacetic acid (5-HIAA) and serum chromogranin $\mathrm{A}(\mathrm{CgA})$ are the most useful initial tests for suspected NET disease (6). Increased serum levels of carcinoembryonic antigen (CEA) may indicate adenocarcinoma (35). No equivalent biochemical markers exist for sarcomas/GISTs and NHL.

Endoscopy. Upper GI endoscopy allows identification of lesions to the level of the ligament of Treitz, and colonoscopy can detect and identify tumors as proximal as the terminal ileum. The majority of the SI is therefore beyond endoscopic surveillance and requires capsule endoscopy (CE) or double balloon enteroscopy (DBE) $(36,37)$. CE provides a useful method for the non-invasive evaluation of patients with abdominal pain or obscure gastointestinal bleeding. Its main disadvantage is the inablity to sample, and the investigation is contraindicated if intestinal obstruction is suspected. DBE provides access to for tissue sampling. However, the procedure is difficult, prolonged, often uncomfortable and requires considerable training. Endoscopic ultrasound (EUS) 

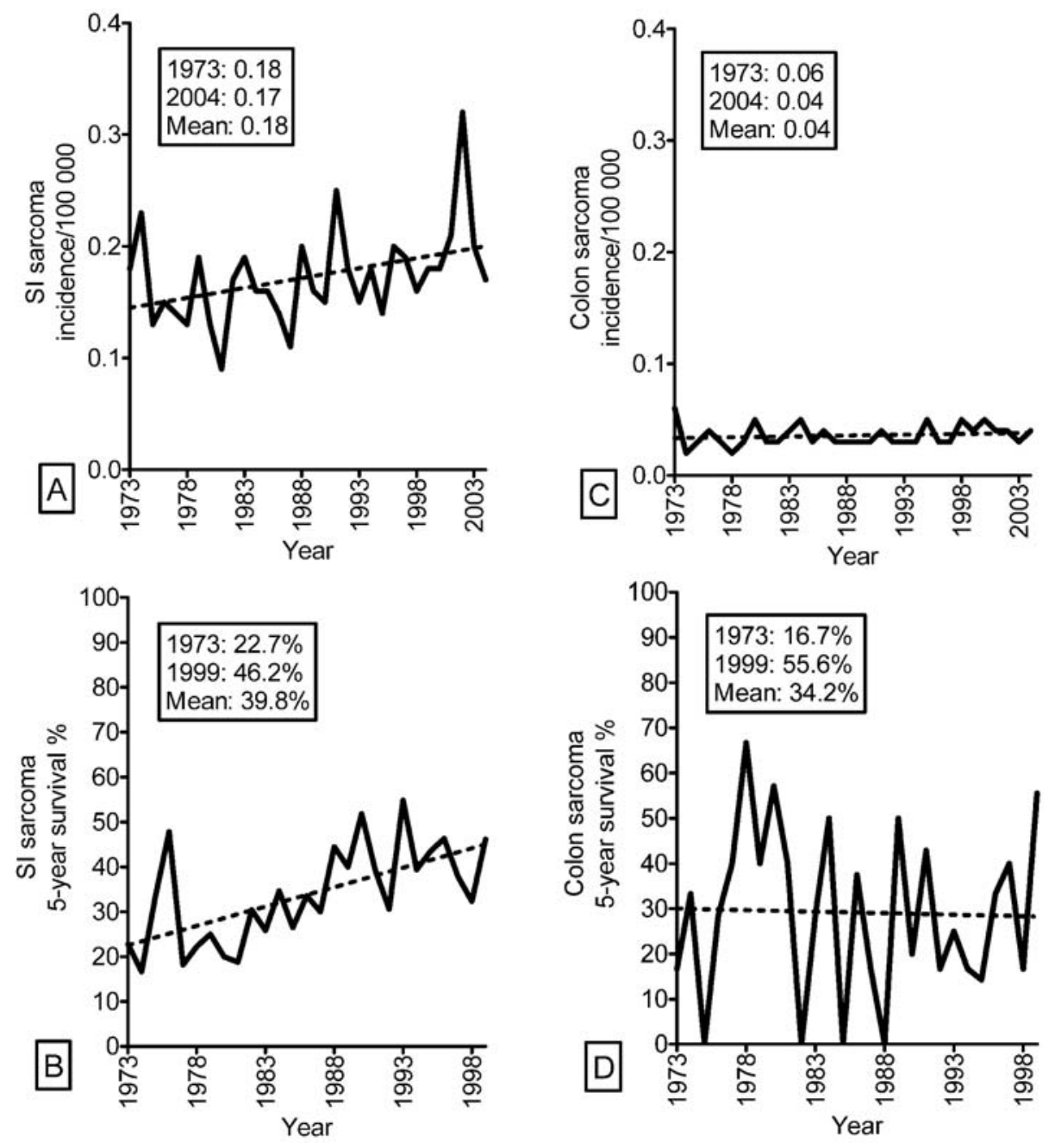

Figure 5. A and B, Incidence and 5-year survival of small intestinal (SI) sarcomas. C and D, Incidence and 5-year survival of colon sarcomas. Dotted lines represent regression curves.

is especially useful in the diagnosis of GISTs. Even small tumors are readily seen on EUS examination, where they characteristically appear as masses embedded within the five-layered wall structure (38). EUS-fine needle aspiration allows tissue samples to be obtained for histopathological diagnosis.

Radiology. Radiological imaging including computer tomography (CT) and magnetic resonance imaging (MRI) are useful in diagnosis, evaluation of spread and response to treatment. Multidetector row CT (MDCT) provides high-resolution imaging and precise delineation of tumor pathology, and its complications including obstruction, involvement of adjacent viscera and metastases (39). MDCT and MDMRI imaging can be combined with traditional techniques such enteroclysis. In a study of 219 patients with suspected SI neoplasia, contrast- and water-enhanced MDCT enteroclysis had an overall accuracy of $84.7 \%$ for identification of SI neoplasia (40).

Nuclear imaging techniques. Tumor-specific radiolabeled somatostatin (SST) receptor analogs are sensitive $(\sim 90 \%)$ in identifying NETs and their metastases (41). Positron emission tomography (PET) detects the accumulation of radiolabeled biological substances such as ${ }^{18} \mathrm{~F}$-fluorodeoxyglucose (FDG) or the radiolabeled precursor of serotonin synthesis, ${ }^{11} \mathrm{C}-5-$ hydroxytryptophan. Combinations of SRS or PET with CT or MRI imaging systems are especially effective as together they produce a particularly high sensitivity (96-100\%) for NET detection $(42,43)$. In a retrospective study of 172 patients with lymphoma, FDG-PET detected disease in at least one site in 161 patients $(94 \%)$ (44). The role of FDG-PET is limited for staging of sarcomas/GISTs because of the low rate of extra-abdominal tumor involvement and lower sensitivity than $\mathrm{CT}$, but may be valuable for the detection of Imatinibresponse in KIT-positive tumors as early as one week after starting therapy (45).

Histopathology. The diagnosis of NETs is supported by morphological features and immunohistochemical (IHC) for $\mathrm{CgA}$, synaptophysin, serotonin, and neuron specific enolase (NSE) (7). Since benign hyperplasia of gastrointestinal lymphoid tissue is relatively common, the morphological diagnosis of lymphoma may be challenging. IHC for B-cell 
markers (CD20) and T-cell markers (CD43) allows recognition of benign lymphoid hyperplasia. Specific IHC markers for lymphoma are leukocyte common antigen (LCA), and antibodies to filament proteins such as vimentin, keratin and desmin.

The precise diagnosis of GIST requires IHC evidence of KIT (CD117) expression. However, KIT expression can be absent in $\leq 15 \%$ of GISTs, and it has been proposed that a novel monoclonal antibody DOG1 (discovered on GIST1) might be a useful diagnostic marker to identify individuals who may benefit from targeted therapy such as Imatinib (46).

Invasive techniques. A pre-laparotomy laparoscopic examination may be helpful for staging, and can reveal any serosal infiltration, retroperitoneal fixation, lymph node involvement, and metastases.

\section{Treatment}

NETs. The only curative treatment for GI-NETs is radical surgical resection. However, small, solitary non-invasive lesions in the colon and duodenum may be treated by endoscopic local resection $(47,48)$. Surgery alone or in combination with other treatment options may be used as palliative treatment in unresectable disease, and has been shown to increase median survival, decrease tumor burden, facilitate symptom control, and prevent complications (49). Small appendiceal NETs may be managed by a simple appendectomy. However, tumors that are larger than $2 \mathrm{~cm}$ in size exhibit mesoappendiceal invasion, vascular invasion, high mitotic activity, or the presence of non-neuroendocrine cell elements (mucin) and specific adverse molecular markers have a significant risk of metastatic spread (30-60\%), and as such, warrant an extended right hemicolectomy (50). Colon NETs have a poor prognosis and should be managed as if adenocarcinomas of the colon utilizing en bloc resection.

Symptomatology (particularly flushing and diarrhea) is best controlled with long-acting SST analogs. Such agents, although generally well-tolerated, have little in vivo inhibitory effect on tumor growth though may have some efficacy in generating tumor stability (51). Interferon (IFN) $-\alpha,-\gamma$, and human leukocyte IFN have some utility in the control of symptoms, however, the onset of the effective symptomatic response is delayed and adverse-effects are common. The response rates for single agent chemotherapy such as doxorubicin, 5-fluorouracil (5-FU), and streptozotocin are 5-10\% (51). Combination chemotherapy has slightly better response rates ranging from 20 to $30 \%$, but the results are overall poor (51). There is general consensus that 'traditional' chemotherapeutic agents are not recommended for NET treatment, with the exception of pancreatic NETs and poorly differentiated neuroendocrine carcinomas (52). New generation anti-tumor treatments such as Imatinib have been shown to actively inhibit NETs in vitro, but in vivo have been disappointing (6).

Peptide receptor radionuclide therapy (PRRT) using of either ${ }^{90} y$ ttrium or ${ }^{177}$ lutetium radionuclides linked to an SST analog enables SST receptor-expressing tumor cells to be selectively targeted. The most effective PRRT currently available for treatment of metastatic GI-NETs is ${ }^{177} \mathrm{Lu}$ bound to the SST analog DOTA ${ }^{0} \mathrm{Tyr}^{3}$ octreotate. In uncontrolled studies, ${ }^{177} \mathrm{Lu}-\mathrm{DOTA}{ }^{0} \mathrm{Tyr}^{3}$ octreotate has been reported to produce tumor responses in $35 \%$, and tumor stabilization in $80-90 \%$ of NETs (53).

Surgical excision, lobectomy or ablative techniques (hepatic artery embolization, cryoablation and radio frequency ablation) can be utilized to reduce tumor load in NETs with hepatic metastases. This is associated with a clinical symptomatic improvement and may extend 5-year survival (54). Liver transplantation has been utilized with success in a minority of patients (55).

SI and appendix adenocarcinomas. The only curative treatment is complete surgical resection but this is often impossible due to tardy diagnosis and local extension and extensive metastasis (56). Jejunal and ileal adenocarcinomas should be aggressively treated with en bloc or segmental resection and primary anastomosis, and pancreaticoduodenectomy considered for duodenal tumors $>1 \mathrm{~cm}$ in size, which have spread beyond the muscularis mucosa or have evidence of aggressive histological criteria (56). Appendix tumors should be managed by right hemicolectomy. Routine oophorectomy is also indicated as ovarian metastases are evident $>50 \%$ of patients with appendiceal adenocarcinomas (57). Histological staging and adjuvant therapy for appendiceal adenocarcinomas are similar to colon adenocarcinomas (57). An overall increased survival for SI adenocarcinoma with combination treatment with chemotherapy and surgery has been noted, however, further randomized controlled trials to evaluate the effectiveness of adjuvant chemotherapy are required (58). Chemotherapeutic regimens usually consist of 5-FU, either alone or in combination with a variety of other agents including doxorubicin, cisplatin, mitomycin $\mathrm{C}$, and cyclophosphamide.

Lymphomas. The management of primary GI tract NHL is debated although surgical exploration is initially warranted for correct diagnosis and staging (59). Further treatment is based on the histological subtype. In a Japanese study of 96 primary intestinal lymphomas, $47 \%$ were treated with surgery alone, $16 \%$ chemotherapy or radiotherapy, $35 \%$ chemotherapy plus radiotherapy, and $2 \%$ received no treatment (60). Patients who received non-surgical treatment showed a better overall survival than those treated by surgery, but event-free survival did not differ between two groups.

In general, rapidly proliferating tumors, such as MALT associated diffuse large B-cell lymphomas, are most chemosensitive and thus have the best overall cure rates. In a prospective study of 40 diffuse large B-cell lymphomas who all received primary surgical resection with lymph node dissection and post-operative CHOP (cyclophosphamide, hydroxydaunorubicin, oncovin, and prednisolone) chemotherapy, the overall 5-year survival rate was $89 \%$ (61). Slower growing, indolent tumors, like follicular lymphomas and mantle cell, are frequently resistant to chemotherapy and in many cases chemotherapy will not alter the prognosis (62). In a study of 25 patients with primary intestinal follicular lymphoma, 7 received no treatment, and 18 received therapy at diagnosis (24). Among these, complete surgical resection alone was undertaken in 4 cases, chemotherapy alone in 10 , complete surgical resection plus chemotherapy in 2 , 
and chemotherapy plus radiotherapy in 2. After follow-up of 34 months, 22 out of 25 patients were still alive (11 complete remission, 6 partial remission, 3 stabile disease, and 2 progressive).

T-cell lymphomas are early to disseminate and complete surgical resection is usually not feasible. In a prospective, multicentre study of 35 patients treated with six cycles of CHOP, the cumulative 2-year survival was only $28 \%$ (63). In another study, 4 patients with ETL received high-dose chemotherapy followed by autologous stem cell transplantation. One had ongoing complete remission 32 months after transplantation while 3 died from relapse within few months (64).

Sarcomas and GISTs. The primary aim should be complete surgical resection with negative margins avoiding the occurrence of tumor spillage. Localized GISTs should be removed en bloc, respecting a possible pseudo capsule and avoiding intraperitoneal dissemination. Adjacent organs adherent to the tumor should also be resected en bloc (65). Medical treatment with Imatinib is indicated in unresectable, metastatic or recurrent GISTs, with the dose adjusted according to response (65). Imatinib treatment can initially cause hemorrhage, edema and myxoid degeneration, resulting in a paradoxical increase in tumor size. The majority ( 70-90\%) of GISTs respond to Imatinib or exhibit disease stabilization with a median duration of response of 2-3 years (66). Secondline therapy requires consideration as $\sim 10 \%$ of patients with advanced GIST exhibit primary resistance to the drug and $\sim 20 \%$ develop secondary resistance (67). Some may benefit from repeat surgical excision of focal GIST progression (66), while others can be treated with Sunitinib, a selective multi targeted TKI. In 312 patients resistant to Imatinib, time to progression was increased from 6.4 weeks in placebo-treated controls to 27.3 weeks in the Sunitinib group (68). Leiomyosarcomas are resistant to both radio and chemotherapy. Surgical resection involving both the tumor and adjacent mesentery offers the only potential cure.

\section{Summary}

Neoplasia of the SI, appendix and colon (except adenocarcinomas) are relatively uncommon but represent an important group of tumors, due to their poor prognosis secondary to late diagnosis, and resistance to conventional cancer therapy. Over the last 30 years, their overall incidence has increased 2.2-fold (1.53-3.36). The advent of novel imaging and capsule endoscopy techniques has improved the diagnosis. Similarly, promising therapeutic data in some subgroups are encouraging although the majority are still diagnosed late and targeted effective therapy is lacking.

Conventional chemotherapy regimens retain some role as adjuvant therapy for adenocarcinomas and subgroups of lymphomas, while the effect on NETs usually is minimal. Advances in the molecular biology of carcinogenesis have led to the development of novel chemotherapeutic agents targeting growth factor signaling. Imatinib, as an inhibitor of the KIT kinases, has revolutionized the treatment of GISTs, and PRRT is a promising new tumor targeted radiotherapy modality for NETs.
Increased awareness and early diagnosis in combination with the development new and more efficient therapy remains the key to improving survival for this relatively rare but steadily increasing group of tumors.

\section{Acknowledgements}

This study was supported by the Norwegian Research Council, Mjålands legacy, Caroline Musæus Aarvolds legacy, Bruggeman Medical Foundation.

\section{References}

1. Liszka L, Zielinska-Pajak E, Pajak J, Golka D and Huszno J: Coexistence of gastrointestinal stromal tumors with other neoplasms. J Gastroenterol 42: 641-649, 2007.

2. Roberts MC, Millikan RC, Galanko JA, Martin C and Sandler RS: Constipation, laxative use, and colon cancer in a North Carolina population. Am J Gastroenterol 98: 857-864, 2003.

3. Ciresi DL and Scholten DJ: The continuing clinical dilemma of primary tumors of the small intestine. Am Surg 61: 698-702, 1995.

4. Grunkemeier GL, Anderson RP and Starr A: Actuarial and actual analysis of surgical results: empirical validation. Ann Thorac Surg 71: 1885-1887, 2001.

5. Oberndorfer S: Karzinoide tumoren des dunndarms. Frankf Z Pathol 1: 426-423, 1907.

6. Gustafsson BI, Kidd M and Modlin IM: Neuroendocrine tumors of the diffuse neuroendocrine system. Curr Opin Oncol 20: 1-12, 2008

7. Modlin IM, Kidd M, Latich I, Zikusoka MN and Shapiro MD: Current status of gastrointestinal carcinoids. Gastroenterology 128: 1717-1751, 2005.

8. The US National Cancer Institute, Surveillance Epidemiology and End Results (SEER) data base, 1973-2004, http://seer. cancer.gov/. 2007

9. Donow C, Pipeleers-Marichal M, Schroder S, Stamm B, Heitz PU and Kloppel G: Surgical pathology of gastrinoma. Site, size, multicentricity, association with multiple endocrine neoplasia type 1, and malignancy. Cancer 68: 1329-1334, 1991.

10. House MG, Yeo CJ and Schulick RD: Periampullary pancreatic somatostatinoma. Ann Surg Oncol 9: 869-874, 2002.

11. Hoffmann KM, Furukawa M and Jensen RT: Duodenal neuroendocrine tumors: classification, functional syndromes, diagnosis and medical treatment. Best Pract Res Clin Gastroenterol 19: 675-697, 2005

12. Zar N, Garmo H, Holmberg L, Rastad J and Hellman P: Longterm survival of patients with small intestinal carcinoid tumors. World J Surg 28: 1163-1168, 2004.

13. Dumper J, Mackenzie S, Mitchell P, Sutherland F, Quan ML and Mew D: Complications of Meckel's diverticula in adults. Can J Surg 49: 353-357, 2006.

14. Anderson DJ: Carcinoid tumor in Meckel's diverticulum: laparoscopic treatment and review of the literature. J Am Osteopath Assoc 100: 432-434, 2000.

15. Pahlavan PS and Kanthan R: Goblet cell carcinoid of the appendix. World J Surg Oncol 3: 36, 2005.

16. Howe JR, Karnell LH, Menck HR and Scott-Conner C: The American College of Surgeons Commission on Cancer and the American Cancer Society. Adenocarcinoma of the small bowel: review of the National Cancer Data Base, 1985-1995. Cancer 86: 2693-2706, 1999.

17. Verma D and Stroehlein JR: Adenocarcinoma of the small bowel: a 60-year perspective derived from M.D. Anderson Cancer Center Tumor Registry. Am J Gastroenterol 101: 1647-1654, 2006.

18. Dabaja BS, Suki D, Pro B, Bonnen M and Ajani J: Adenocarcinoma of the small bowel: presentation, prognostic factors, and outcome of 217 patients. Cancer 101: 518-526, 2004.

19. Solem CA, Harmsen WS, Zinsmeister AR and Loftus EV Jr: Small intestinal adenocarcinoma in Crohn's disease: a casecontrol study. Inflamm Bowel Dis 10: 32-35, 2004.

20. Green PH, Fleischauer AT, Bhagat G, Goyal R, Jabri B and Neugut AI: Risk of malignancy in patients with celiac disease. Am J Med 115: 191-195, 2003.

21. Xie J and Itzkowitz SH: Cancer in inflammatory bowel disease. World J Gastroenterol 14: 378-389, 2008. 
22. Burke CA, Santisi J, Church J and Levinthal G: The utility of capsule endoscopy small bowel surveillance in patients with polyposis. Am J Gastroenterol 100: 1498-1502, 2005.

23. Ito H, Osteen RT, Bleday R, Zinner MJ, Ashley SW and Whang EE: Appendiceal adenocarcinoma: long-term outcomes after surgical therapy. Dis Colon Rectum 47: 474-480, 2004.

24. Damaj G, Verkarre V, Delmer A, et al: Primary follicular lymphoma of the gastrointestinal tract: a study of 25 cases and a literature review. Ann Oncol 14: 623-629, 2003.

25. Grogg KL, Miller RF and Dogan A: HIV infection and lymphoma. J Clin Pathol 60: 1365-1372, 2007.

26. Yin L, Chen CQ, Peng CH, et al: Primary small-bowel nonHodgkin's lymphoma: a study of clinical features, pathology, management and prognosis. J Int Med Res 35: 406-415, 2007.

27. Isaacson PG and Du MQ: Gastrointestinal lymphoma: where morphology meets molecular biology. J Pathol 205: 255-274, 2005.

28. Romaguera $\mathrm{J}$ and Hagemeister FB: Lymphoma of the colon. Curr Opin Gastroenterol 21: 80-84, 2005.

29. Wong MT and Eu KW: Primary colorectal lymphomas. Colorectal Dis 8: 586-591, 2006.

30. Zighelboim J and Larson MV: Primary colonic lymphoma. Clinical presentation, histopathologic features, and outcome with combination chemotherapy. J Clin Gastroenterol 18: 291-297, 1994.

31. Romaguera JE, Medeiros LJ, Hagemeister FB, et al: Frequency of gastrointestinal involvement and its clinical significance in mantle cell lymphoma. Cancer 97: 586-591, 2003.

32. Bumming P, Ahlman H, Andersson J, Meis-Kindblom JM, Kindblom LG and Nilsson B: Population-based study of the diagnosis and treatment of gastrointestinal stromal tumours. Br J Surg 93: 836-843, 2006.

33. Nilsson B, Bumming P, Meis-Kindblom JM, et al: Gastrointestinal stromal tumors: the incidence, prevalence, clinical course, and prognostication in the preimatinib mesylate era - a population-based study in western Sweden. Cancer 103: 821-829, 2005.

34. Perez EA, Gutierrez JC, Jin X, et al: Surgical outcomes of gastrointestinal sarcoma including gastrointestinal stromal tumors: a population-based examination. J Gastrointest Surg 11: 114-125, 2007.

35. Modlin IM, Latich I, Zikusoka M, Kidd M, Eick G and Chan AK: Gastrointestinal carcinoids: the evolution of diagnostic strategies. J Clin Gastroenterol 40: 572-582, 2006.

36. Yamaguchi T, Manabe N, Tanaka S, et al: Multiple carcinoid tumors of the ileum preoperatively diagnosed by enteroscopy with the double-balloon technique. Gastrointest Endosc 62: 315-318, 2005.

37. Cobrin GM, Pittman RH and Lewis BS: Increased diagnostic yield of small bowel tumors with capsule endoscopy. Cancer 107: 22-27, 2006.

38. Nickl NJ: Gastrointestinal stromal tumors: new progress, new questions. Curr Opin Gastroenterol 20: 482-487, 2004.

39. Ramachandran I, Sinha R, Rajesh A, Verma R and Maglinte DD: Multidetector row CT of small bowel tumours. Clin Radiol 62: 607-614, 2007.

40. Pilleul F, Penigaud M, Milot L, Saurin JC, Chayvialle JA and Valette PJ: Possible small-bowel neoplasms: contrast-enhanced and water-enhanced multidetector CT enteroclysis. Radiology 241: 796-801, 2006.

41. Rodrigues M, Traub-Weidinger T, Li S, Ibi B and Virgolini I: Comparison of 111In-DOTA-DPhe1-Tyr3-octreotide and 111In-DOTA-lanreotide scintigraphy and dosimetry in patients with neuroendocrine tumours. Eur J Nucl Med Mol Imaging 33: 532-540, 2006.

42. Koopmans KP, De Vries EG, Kema IP, et al: Staging of carcinoid tumours with 18F-DOPA PET: a prospective, diagnostic accuracy study. Lancet Oncol 7: 728-734, 2006.

43. Seemann MD: Detection of metastases from gastrointestinal neuroendocrine tumors: prospective comparison of 18F-TOCA PET, triple-phase CT, and PET/CT. Technol Cancer Res Treat 6: 213-220, 2007

44. Elstrom R, Guan L, Baker G, et al: Utility of FDG-PET scanning in lymphoma by WHO classification. Blood 101: 3875-3876, 2003.

45. Jager PL, Gietema JA and van der Graaf WT: Imatinib mesylate for the treatment of gastrointestinal stromal tumours: best monitored with FDG PET. Nucl Med Commun 25: 433-438, 2004.
46. Espinosa I, Lee CH, Kim MK, et al: A novel monoclonal antibody against DOG1 is a sensitive and specific marker for gastrointestinal stromal tumors. Am J Surg Pathol 32: 210-218, 2008

47. Itoi $\mathrm{T}$, Sofuni A, Itokawa $\mathrm{F}$, Tsuchiya $\mathrm{T}$, Kurihara $\mathrm{T}$ and Moriyasu F: Endoscopic resection of carcinoid of the minor duodenal papilla. World J Gastroenterol 13: 3763-3764, 2007.

48. Merg A, Wirtzfeld D, Wang J, Cheney R, Dunn KB and Rajput A: Viability of endoscopic and excisional treatment of early rectal carcinoids. J Gastrointest Surg 11: 893-897, 2007.

49. Kerstrom G, Hellman P and Hessman O: Midgut carcinoid tumours: surgical treatment and prognosis. Best Pract Res Clin Gastroenterol 19: 717-728, 2005.

50. Stinner B and Rothmund M: Neuroendocrine tumours (carcinoids) of the appendix. Best Pract Res Clin Gastroenterol 19: 729-738, 2005.

51. Modlin IM, Latich I, Kidd M, Zikusoka M and Eick G: Therapeutic options for gastrointestinal carcinoids. Clin Gastroenterol Hepatol 4: 526-547, 2006.

52. Eriksson B, Kloppel G, Krenning E, et al: Consensus guidelines for the management of patients with digestive neuroendocrine tumors - well-differentiated jejunal-ileal tumor/carcinoma. Neuroendocrinology 87: 8-19, 2008.

53. Kwekkeboom DJ, Mueller-Brand J, Paganelli G, et al: Overview of results of peptide receptor radionuclide therapy with 3 radiolabeled somatostatin analogs. J Nucl Med 46 (Suppl. 1): S62-S66, 2005.

54. Sarmiento JM, Heywood G, Rubin J, Ilstrup DM, Nagorney DM and Que FG: Surgical treatment of neuroendocrine metastases to the liver: a plea for resection to increase survival. J Am Coll Surg 197: 29-37, 2003.

55. Mazzaferro V, Pulvirenti A and Coppa J: Neuroendocrine tumors metastatic to the liver: how to select patients for liver transplantation? J Hepatol 47: 460-466, 2007.

56. Wu TJ, Yeh CN, Chao TC, Jan YY and Chen MF: Prognostic factors of primary small bowel adenocarcinoma: univariate and multivariate analysis. World J Surg 30: 391-399, 2006.

57. O'Donnell ME, Badger SA, Beattie GC, Carson J and Garstin WI: Malignant neoplasms of the appendix. Int J Colorectal Dis 22: 1239-1248, 2007.

58. Singhal N and Singhal D: Adjuvant chemotherapy for small intestine adenocarcinoma. Cochrane Database Syst Rev 3: CD005202, 2007.

59. Talamonti MS, Goetz LH, Rao S and Joehl RJ: Primary cancers of the small bowel: analysis of prognostic factors and results of surgical management. Arch Surg 137: 564-570, 2002.

60. Nakamura S, Matsumoto T, Iida M, Yao T and Tsuneyoshi M: Primary gastrointestinal lymphoma in Japan: a clinicopathologic analysis of 455 patients with special reference to its time trends. Cancer 97: 2462-2473, 2003.

61. Lee J, Kim WS, Kim K, et al: Prospective clinical study of surgical resection followed by $\mathrm{CHOP}$ in localized intestinal diffuse large B cell lymphoma. Leuk Res 31: 359-364, 2007.

62. Brandt L, Kimby E, Nygren P and Glimelius B: A systematic overview of chemotherapy effects in indolent non-Hodgkin's lymphoma. Acta Oncol 40: 213-223, 2001.

63. Daum S, Ullrich R, Heise W, et al: Intestinal non-Hodgkin's lymphoma: a multicenter prospective clinical study from the German Study Group on Intestinal Non-Hodgkin's Lymphoma. J Clin Oncol 21: 2740-2746, 2003.

64. Al-Toma A, Verbeek WH, Visser OJ, et al: Disappointing outcome of autologous stem cell transplantation for enteropathy-associated T-cell lymphoma. Dig Liver Dis 39: 634-641, 2007.

65. Blay JY, Bonvalot $\mathrm{S}$, Casali $\mathrm{P}$, et al: Consensus meeting for the management of gastrointestinal stromal tumors. Report of the GIST Consensus Conference of 20-21 March 2004, under the auspices of ESMO. Ann Oncol 16: 566-578, 2005.

66. Joensuu H: Second line therapies for the treatment of gastrointestinal stromal tumor. Curr Opin Oncol 19: 353-358, 2007.

67. Delbaldo C, Chatelut E, Re M, et al: Pharmacokinetic-pharmacodynamic relationships of imatinib and its main metabolite in patients with advanced gastrointestinal stromal tumors. Clin Cancer Res 12: 6073-6078, 2006.

68. Demetri GD, van Oosterom AT, Garrett CR, et al: Efficacy and safety of sunitinib in patients with advanced gastrointestinal stromal tumour after failure of imatinib: a randomised controlled trial. Lancet 368: 1329-1338, 2006. 